\title{
La cytogénétique des animaux d'élevage
}

\author{
Paul Popescu
}

\section{Société Française de Génétique \\ Président \\ E. Moustacchi \\ Vice-présidents \\ P.-H. Gouyon \\ A. Nicolas \\ C. Stoll \\ Secrétaire général \\ R. Motta}

Prière d'adresser toute correspondance au Secrétariat général de la SFG, Roland Motta, Institut de recherches scientifiques sur le cancer, laboratoire de recherches génétiques sur les modèles animaux, 7, rue Guy-Moquet, 94802 Villejuif Cedex, France.

\section{Comité de rédaction}

A. Bernheim

J.-C. Dreyfus

M. Fellous

J. Génermont

F. Minvielle

R. Motta

A. Nicolas

S. Sommer

D. de Vienne

Secrétaire

M.-L. Prunier
Le patrimoine héréditaire d'un être vivant, constitué de quelques 50000 à 100000 gènes est codé dans la molécule d'ADN. Associée à divers types de protéines, la molécule d'ADN, longue chez l'homme, de plus d'un mètre, est contenue dans les chromosomes.

Le nombre et la forme des chromosomes sont toujours les mêmes pour une espèce donnée. Ainsi il y a 8 chromosomes chez la drosophile mais un millier chez certaines espèces d'insectes. Chez les mammifères, leur nombre varie de 6 chez une espèce de cerf à près d'une centaine chez un petit rongeur d'Amérique du Sud. Toutefois, de nombreuses espèces de mammifères, dont l'homme et les primates, possèdent autour de 50 chromosomes. Les paramètres normaux des chromosomes, leur nombre et morphologie, leurs variations ainsi que la localisation des gènes qu'ils contiennent, constituent l'objet d'étude de la cytogénétique.

\section{L'homme : un modèle pour la cytogénétique vétérinaire}

Chez l'homme, la première anomalie chromosomique, la trisomie du chromosome 21, reconnue responsable de la maladie de Down, fut découverte en 1959. Durant la décennie qui suivit, il a été établi une longue liste d'anomalies chromosomiques telles les inversions, les délétions ou les translocations, impliquées dans des malformations diverses, débilité mentale et troubles de fertilité.
Au début des années 1970, plusieurs techniques de marquage chromosomique ont été mises au point. Ces techniques de marquage, appelées aussi techniques de bandes, produisent sur chaque paire de chromosome un dessin constitué d'une succession de bandes claires et sombres, toujours le même pour un chromosome donné. Ces techniques permettent d'apparier les chromosomes homologues et d'identifier les anomalies du nombre ou de la structure des chromosomes. Des études faites sur les avortements spontanés ont montré l'importance majeure des anomalies chromosomiques dans la mortalité embryonnaire. En effet, les anomalies chromosomiques sont responsables, chez la femme, d'environ $50 \%$ des avortements précoces à 10 semaines après la fécondation. La fréquence de telles anomalies est encore plus élevée, environ $75 \%$ dans des cas ou le développement de l'embryon s'arrête plus tôt, 2 à 3 semaines après la fécondation.

Des possibilités nouvelles sont ouvertes pour le diagnostic prénatal avec le dépistage de maladies géniques dues à l'altération d'un gène unique. Près de 3000 maladies génétiques ont été recensées chez l'homme dont certaines, en raison de leur fréquence élevée, posent un grave problème de santé. Pour quelques unes, telles la myopathie de Duchenne, l'hémophilie ou la mucoviscidose, le gène défectueux a été localisé sur les chromosomes et isolé, permettant un diagnostic prénatal dans de nombreuses maladies.

Un autre domaine de la cytogénétique qui s'est beaucoup développé ces dernières années est la carte génique qui 
consiste à localiser précisément chaque gène sur un chromosome. Les techniques utilisées pour la localisation des gènes sur les chromosomes reposaient au début sur l'analyse de la ségrégation de couples de caractères dans des familles ou sur la technique de l'hybridation cellulaire interspécifique. Les progrès récents de l'hybridation in situ avec des sondes d'ADN radioactives ou "froides " ont permis de localiser un nombre important de gènes.

La technique de fécondation in vitro hétérospécifique mise au point ces dernières années, a ouvert la voie à la cytogénétique du gamète mâle. Cela revêt un intérêt particulier pour l'étude du caryotype du spermatozoïde des individus normaux, mais aussi, des individus porteurs d'une anomalie chromosomique de structure à l'état équilibré.

L'étude cytogénétique du gamète humain femelle a, elle aussi, beaucoup progressé récemment, grâce au développement de la fécondation in vitro. Le tiers environ d'ovocytes recueillis pour la fécondation in vitro et qui restent infécondés ont permis de connaître le rôle du gamète femelle dans la genèse des anomalies chromosomiques de l'œuf fécondé. Ce domaine nouveau de la cytogénétique humaine a été récemment exposé dans un excellent article publié dans médecine/sciences [1].

\section{La cytogénétique des animaux d'élevage}

Les progrès techniques réalisés en cytogénétique humaine ont été progressivement appliqués aux mammifères et en particulier aux espèces d'élevage. Les premières anomalies de la structure chromosomique ont été découvertes en 1964: une translocation réciproque chez le porc et une fusion robertsonienne chez le taureau [2]. La mise en évidence ultérieure de leurs conséquences néfastes sur la fertilité et la prolificité, composantes majeures de la productivité numérique [3] a éveillé l'intérêt des chercheurs pour la cytogénétique des animaux domestiques.

\begin{tabular}{|c|c|c|c|c|c|c|}
\hline \multicolumn{7}{|c|}{$\begin{array}{c}\text { Tableau I } \\
\text { NOMBRE CHROMOSOMIQUE DES ANIMAUX D'ÉLEVAGE } \\
\text { ET DE QUELQUES ESPĖCES SAUVAGES APPARENTÉES }\end{array}$} \\
\hline \multirow{2}{*}{ Espèces } & \multirow{2}{*}{$2 n$} & \multirow{2}{*}{ NF } & \multicolumn{2}{|c|}{ Autosomes } & \multicolumn{2}{|c|}{$\begin{array}{l}\text { Chromosomes } \\
\text { sexuels }\end{array}$} \\
\hline & & & $M+S M$ & $A$ & $x$ & Y \\
\hline $\begin{array}{l}\text { Bos taurus - Bœuf domes- } \\
\text { tique }\end{array}$ & 60 & 62 & 0 & 58 & SM & SM \\
\hline $\begin{array}{l}\text { Bos indicus - Zebu } \\
\text { Bos banteng - Bouf Java- } \\
\text { nais, banteng } \\
\text { Bos grunniens - Yak }\end{array}$ & $\begin{array}{l}60 \\
60\end{array}$ & $\begin{array}{l}62 \\
62\end{array}$ & $\begin{array}{l}0 \\
0\end{array}$ & $\begin{array}{l}58 \\
58\end{array}$ & $\begin{array}{l}\text { SM } \\
\text { SM }\end{array}$ & $\begin{array}{l}\text { A } \\
\text { SM } \\
\text { SM }\end{array}$ \\
\hline $\begin{array}{l}\text { Bison bonasus - Bison } \\
\text { européen } \\
\text { Bison bison - Bison amé- } \\
\text { ricain }\end{array}$ & $\begin{array}{l}60 \\
60\end{array}$ & $\begin{array}{l}62 \\
62\end{array}$ & $\begin{array}{l}0 \\
0\end{array}$ & $\begin{array}{l}58 \\
58\end{array}$ & $\begin{array}{l}\text { SM } \\
\text { SM }\end{array}$ & $\begin{array}{c}\text { SM } \\
\text { A }\end{array}$ \\
\hline $\begin{array}{l}\text { Bubalus bubalis - Buffle } \\
\text { d'eau } \\
\text { Syncerus caffer caffer - } \\
\text { Buffle africain }\end{array}$ & 50 & 60 & 10 & 40 & A & A \\
\hline $\begin{array}{l}\text { Ovis aries - Mouton } \\
\text { domestique } \\
\text { Ovis musimon - Mouflon } \\
\text { Capra hircus - Chèvre }\end{array}$ & $\begin{array}{l}54 \\
54 \\
60\end{array}$ & $\begin{array}{l}60 \\
60 \\
60\end{array}$ & $\begin{array}{l}6 \\
6 \\
0\end{array}$ & $\begin{array}{l}52 \\
52 \\
58\end{array}$ & $\begin{array}{l}A \\
A \\
A\end{array}$ & $\begin{array}{l}\text { SM } \\
\text { SM } \\
\text { SM }\end{array}$ \\
\hline $\begin{array}{l}\text { Sus scrofa domestica } \\
\text { Porc domestique } \\
\text { Sus sacrofa - Sanglier }\end{array}$ & $\begin{array}{l}38 \\
36\end{array}$ & $\begin{array}{l}64 \\
64\end{array}$ & $\begin{array}{l}24 \\
26\end{array}$ & $\begin{array}{l}12 \\
10\end{array}$ & $\begin{array}{l}\text { SM } \\
\text { SM }\end{array}$ & $\begin{array}{l}\mathrm{SM} \\
\mathrm{SM}\end{array}$ \\
\hline $\begin{array}{l}\text { Equus Caballus - Cheval } \\
\text { domestique } \\
\text { Equus przewalskii - Cheval } \\
\text { de Przewalski }\end{array}$ & $\begin{array}{l}64 \\
66\end{array}$ & $\begin{array}{l}88 \\
88\end{array}$ & $\begin{array}{l}26 \\
24\end{array}$ & $\begin{array}{l}36 \\
40\end{array}$ & $\begin{array}{l}\text { SM } \\
\text { SM }\end{array}$ & $\begin{array}{l}A \\
A\end{array}$ \\
\hline
\end{tabular}

$N F=$ nombre fondamental (nombre des bras); $M=$ métacentrique $; S M=$ submétacentrique ; $A$ $=$ acrocentrique.

\section{Le caryotype normal}

A l'exception du porc, les principales espèces de mammiferes domestiques ont un caryotype difficile à étudier en raison du nombre élevé de chromosomes (64 chez le cheval, 60 chez le taureau et la chèvre) et, de plus, de leur morphologie semblable (Tableau I). Le porc lui ne possède que 38 chromoso- mes, ayant des morphologies différentes et, de ce fait, relativement plus faciles à étudier.

Les techniques de coloration classiques utilisées pendant les années 1960, donnaient une image uniforme des chromosomes, sans aucun détail morphologique à l'exception de la position du centromère. Chez des espèces dont tous (ou presque tous) les autosomes sont acrocentriques, seule la taille des 
chromosomes était prise en considération pour apparier les deux homologues et les ranger dans une série décroissante, divisée arbitrairement en plusieurs rangées. Dès le début des années 1970 différentes techniques de marquage ont été adaptées aux chromosomes des espèces domestiques, facilitant l'identification des chromosomes homologues et la mise en évidence des anomalies.

En 1976, lors de la " conférence de Reading " [4], un caryotype en bandes G standard a été proposé pour chaque espèce domestique. En mai 1989, nous avons organisé à Jouy la "deuxième conférence internationale de standardisation des caryotypes des espèces domestiques ". La conférence a réuni une trentaine de chercheurs venus d'Europe, d'Amérique du Nord et du Sud et d'Australie. Dans la publication finale [5], il a été établi une corrélation entre les chromosomes marqués en bandes $\mathrm{G}$ et bandes $\mathrm{R}$ des bovins du mouton et de la chèvre. Pour ces trois espèces ont été proposés des caryotypes standards en bandes $\mathrm{R}$ ainsi que des représentations schématiques en bandes $G$ et $R$. A l'exception du chromosome 9 et de l'X une homologie du dessin des bandes a été établie entre ces trois espèces [6] (figure 1). Cela est très important pour la cartographie des gènes. En effet, si un gène est assigné à un chromosome donné dans l'une des trois espèces, il y a de fortes chances qu'il ait la même localisation chez les deux autres.

La section cheval de la conférence de Jouy a publié séparément le caryotype standard dans Hereditas [7] et le caryotype du porc avait déjà été établi deux ans plus tôt et publié dans la même revue [8].

Anomalies chromosomiques

et leurs conséquences zootechniques chez les bovins

Le bovin domestique est, avec quelque 25000 animaux examinés, l'espèce de mammifère la plus étudiée, après la souris, du point de vue cytogénétique. Cela s'explique par la découverte en 1964 en Suède, de la $\mathrm{m} / \mathrm{s} n^{\circ} 9$ vol. 7, novenbre 91

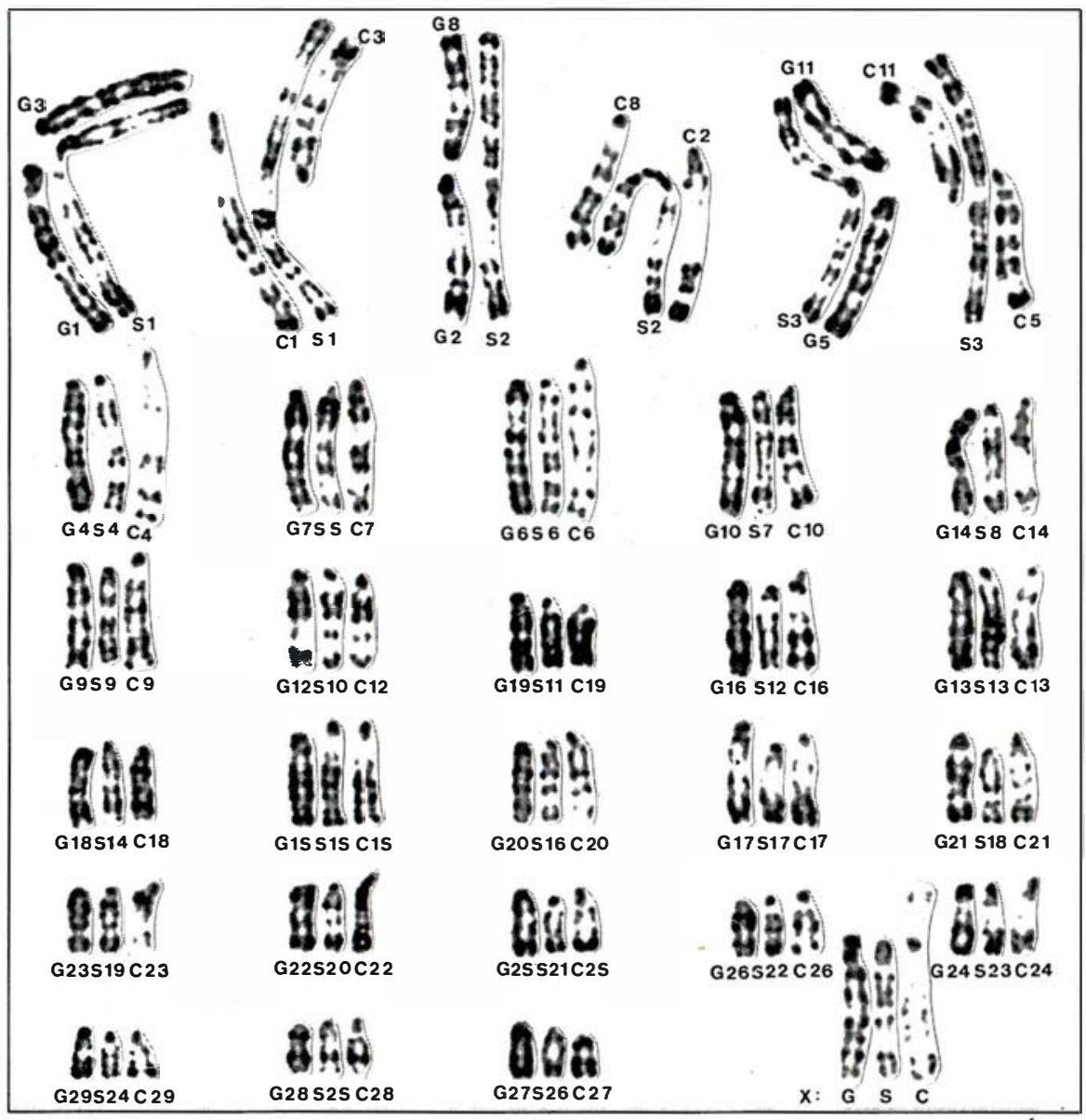

Figure 1. Montage des caryotypes haploïdes en bandes RBG de chèvre (G) mouton (S) et bovin (C). (H. Hayes, laboratoire de cytogénétique de Jouy-en-Josas).

translocation Robertsonienne 1/29 [9] (figure 2) qui a des conséquences néfastes, zootechniques et économiques. En effet, elle réduit la fertilité des femelles porteuses hétérozygotes d'environ $10 \%$. Depuis la première observation de Gustavsson cette anomalie a été retrouvée dans une cinquantaine de races bovines répandues sur les 5 continents[2].

La fréquence des porteurs hétérozygotes de cette anomalie varie d'une race à une autre. Dans certaines races à effectif très réduit telle la Bristish White ou la race Corse elle atteint des fréquences très élevées, respectivement de $60 \%$ et $40 \%$ [2]. Une étude faite en France en race Blonde d'Aquitaine, sur un échantillon représentatif de plus de 2000 génisses, à montré une fréquence d'environ $14 \%$ des hétérozygotes [10].
La réduction de la fertilité des animaux hétérozygotes s'explique par une augmentation de la mortalité embryonnaire, due aux gamètes à caryotype déséquilibré, par défaut ou par excès d'un chromosome. Ces gamètes a caryotype déséquilibré qui gardent leur capacité de fécondation normale, donnent des embryons non viables, monosomiques ou trisomiques, pour l'un ou l'autre des deux chromosomes impliqués dans l'anomalie (figure 3).

Après la découverte de l'anomalie, le ministère suédois de l'Agriculture a estimé qu'en raison de la réduction de la fertilité provoquée par cette anomalie, les éleveurs subissaient chaque année une perte d'environ 2 millions de francs. Une politique d'éradication de cette anomalie a été mise en place en Suède d'abord, et ensuite dans de nombreux pays dont la France. 
La découverte de cette anomalie, et de ses conséquences sur l'élevage a eu un impact considérable sur le développement de la cytogénétique des animaux d'élevage et sur la sélection des reproducteurs.

Les conséquences économiques des anomalies chromosomiques chez le porc

L'anomalie de structure la plus fréquente chez le porc est la translocation réciproque. L'animal porteur d'une telle translocation a un phénotype normal, mais il engendre des portées réduites, généralement d'environ $50 \%$ [2].

En France, le dépistage de ces anomalies est facilité par le Programme National de Gestion Technique des Troupeaux de truies. Ce programme inventorie actuellement environ $60 \%$ des portées nées en France, et classe les verrats selon la taille des portées qu'ils produisent. Le laboratoire de cytogénétique de Jouy, étudie depuis la fin des années 1970, tous les verrats dits "hypoprolifiques" qui engendrent 8 porcelets ou moins, sur un minimum de 6 portées (le nombre moyen des porcelets nés vivants par portées en France est de 10,7). Plus de la moitié de ces verrats ont été trouvés porteurs d'une anomalie chromosomique (figure 5).

L'application d'un modèle de simulation technico-économique "Porsim" a montré qu'un élevage de 42 truies utilisant un verrat porteur d'une translocation qui réduit la taille des portées de moitié subit des pertes considérables de l'ordre de $50 \%$ [11].

Plus récemment $M$. Bonneau [12] a simulé les effets d'une translocation réciproque sur un verrat utilisé dans l'insémination artificielle. Il a trouvé qu'un verrat croisé utilisé dans un centre d'insémination artificielle (IA) pour inséminer 585 truies, provoquerait une perte d'environ $600000 \mathrm{~F}$ par an. L'insémination artificielle, encore très peu répandue en France il y a quelques années, connaît actuellement une expansion sans précédent. Le contrôle cytogénétique de routine de tous les animaux utilisés pour l'insémination IV

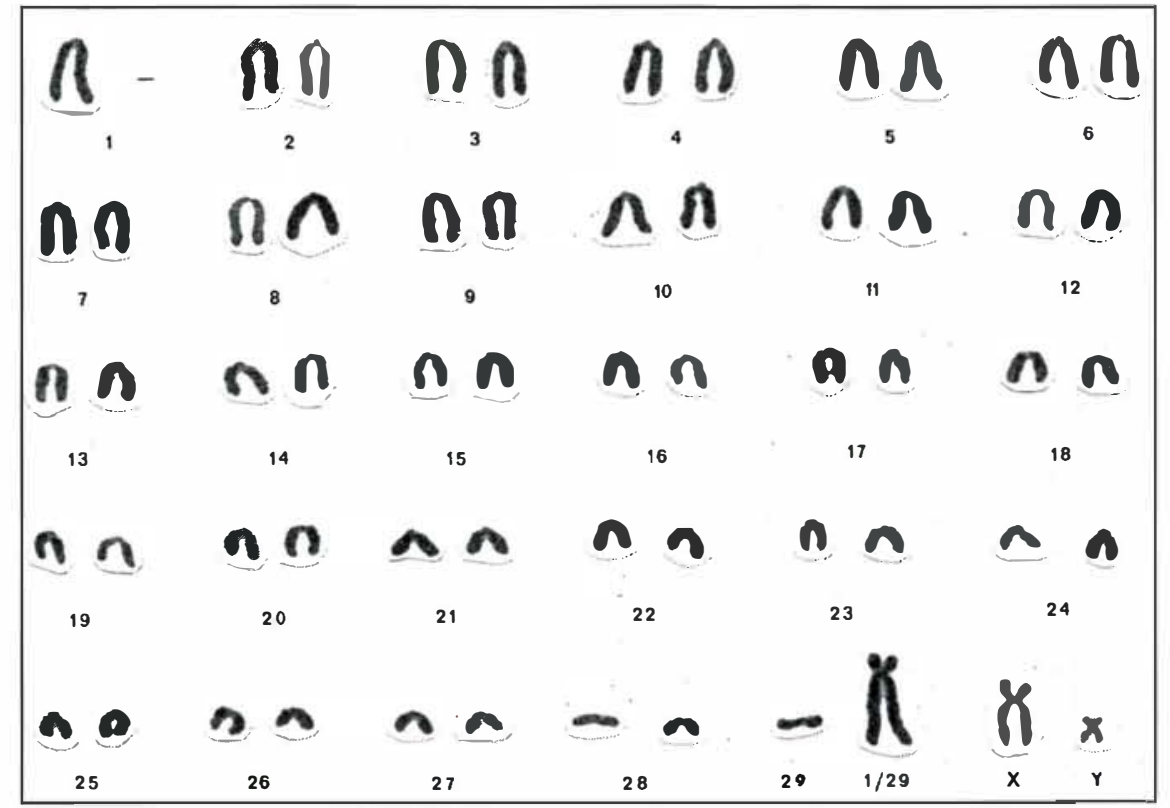

Figure 2. Caryotype d'un bovin porteur de la translocation 1/29.
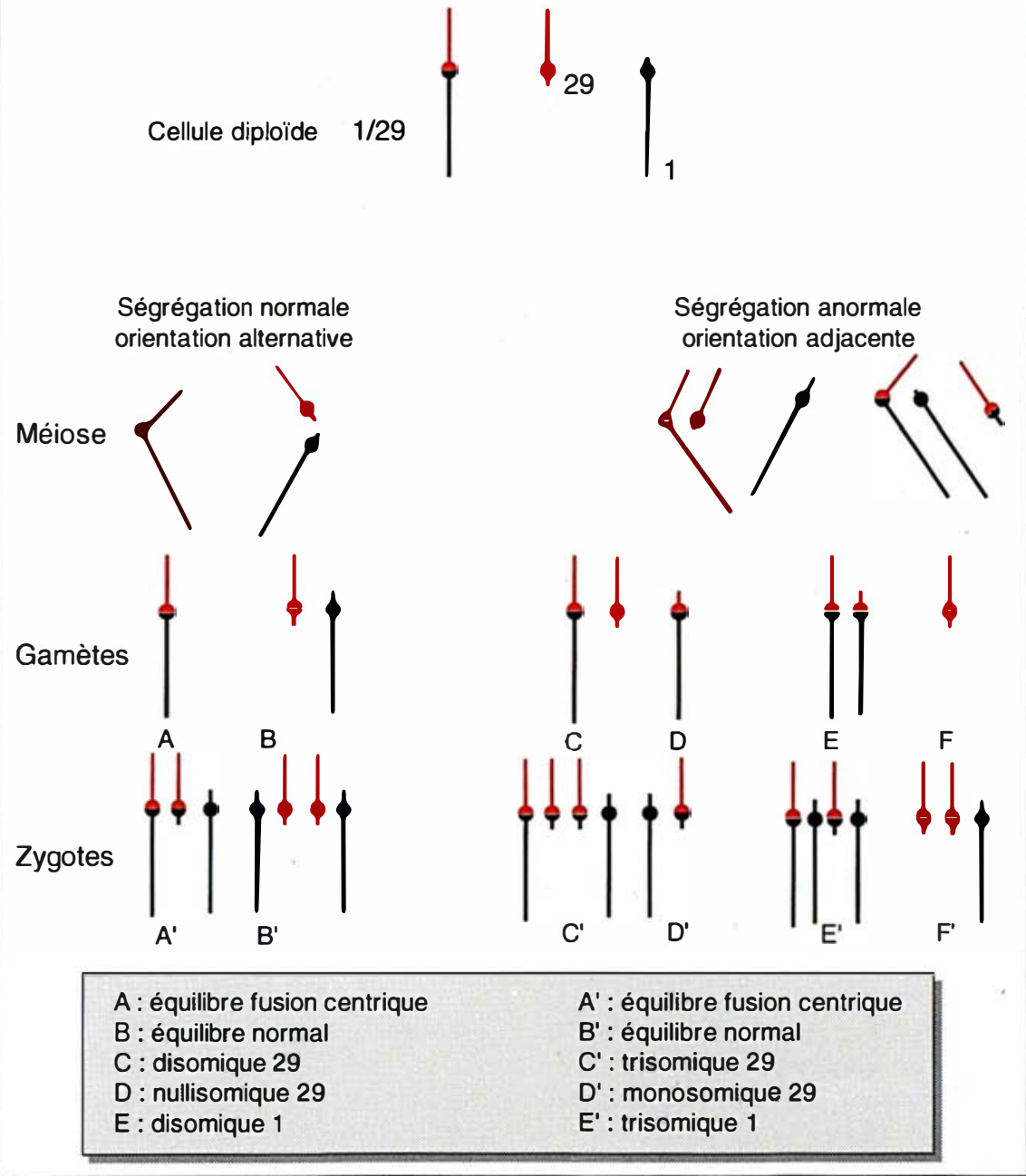

$m / s n^{\circ} 9$ vol. 7 , novembre 91 


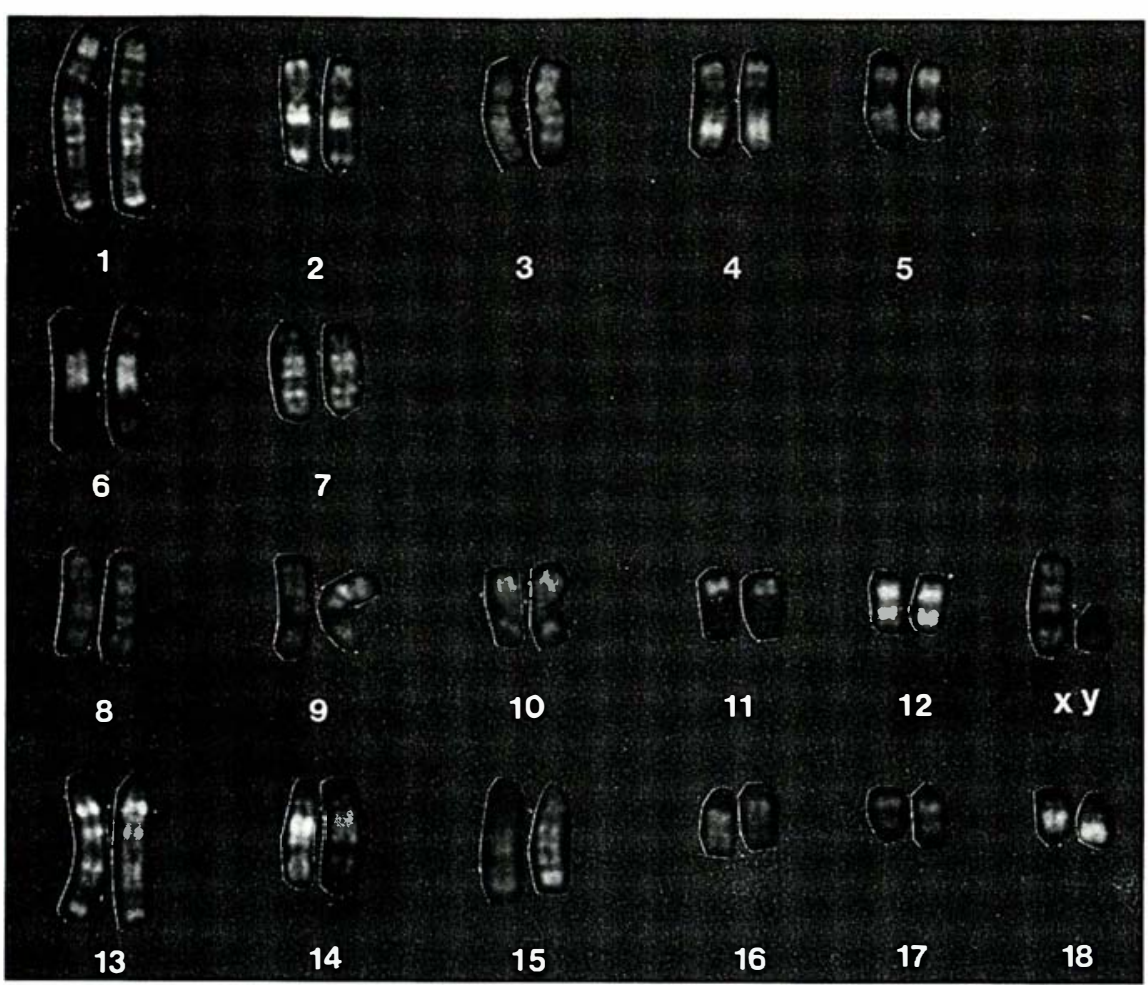

Figure 4. Caryotype de porc normal en bandes RBA (J. Boscher, laboratoire de cytogénétique, INRA, Jouy-en-Josas).

Les chromosomes

des vertébrés inférieurs

Parmi les vertébrés inférieurs tels que les poissons, les batraciens et les reptiles, seuls les poissons ont un grand intérêt zootechnique.

Sur les quelques 20000 espèces différentes de poissons, peu d'espèces ont été étudiées du point de vue cytogénétique. Ces études, bien qu'incomplètes ont montré une grande variation de la taille du génome des poissons. Ainsi, les dipneustes (poissons à poumons) qui ont très peu évolué depuis le dévonien, possèdent un génome trente fois plus grand que celui des mammifères. Cette redondance du génome des poissons est probablement apparue, comme l'a montré Ohno, par la duplication en tandem, au début de leur évolution. Chez certains

4 Figure 3. Les différents types de gamètes et zygotes produits par un animal hétérozygote pour la translocation 1/29. (Daprès [8]).

$\mathrm{m} / \mathrm{s} n^{\circ} 9$ vol. 7 , nocembre 91
C'est pourquoi les vertébrés inférieurs, tels les poissons, peuvent servir de modèle pour la recherche en reproduction animale.

\section{La cytogénétique des oiseaux}

Les oiseaux, comme les reptiles, possèdent des macrochromosomes facilement identifiables et des microchromosomes dont la morphologie n'est pas clairement visible. Les chromosomes sexuels également comme chez les reptiles, sont de type ZW/ZZ, la femelle étant hétérogamétique.

Le nombre chromosomique, chez les différentes espèces d'oiseaux, est génétiquement très élevé et peut atteindre une centaine. La différence de taille entre les macrochromosomes et microchromosomes peut-être très nette ou au contraire, il peut y avoir un passage progressif.

Parmi les oiseaux d'élevage, la poule domestique est l'espèce la plus étudiée. Elle possède 78 chromosomes dont une dizaine de paires sont des macrochromosomes. Les autres étant de très petite taille et sans morphologie définie. Toutefois, dans les mitoses présentant des chromosomes allongés, le nombre de $2 n=78$ est constant.

Les chromosomes de la poule sont obtenus à partir de cultures de lymphocytes ou de fibroblastes.

Plusieurs techniques de marquage chromosomiques appliquées au chromosomes de la poule ont permis l'identification précise d'une dizaine de paires de macrochromosomes.

Le rôle des anomalies chromosomiques sur la mortalité embryonnaire a été surtout étudié sur des cellules de très jeunes embryons. En effet Fechheimer [14] a mis au point au milieu des années soixante dix, une technique qui permet l'examen cytogénétique sur un embryon obtenu après 16 à 18 heures d'incubation. Cette technique a permis d'obtenir des données très fiables, recueillies sur des échantillons très importants, sur la fréquence des différents types d'anomalies chromosomiques et de leurs conséquences sur la mortalité embryonnaire. Ainsi, dans les souches de chair, le chimérisme haploïde/diploïde et la triploïdie pure 
ou en mosaïque, sont les anomalies les plus fréquentes [14]. Les anomalies de la structure chromosomique peuvent être facilement induites chez les poussins juste après l'éclosion, en leur injectant des agents alkylants. Ainsi de nombreuses translocations réciproques et des inversions ont été obtenues. Contrairement à ce qui se passe chez les mammiferes, les oiseaux hétérozygotes pour une translocation réciproque, produisent rarement des gamètes à caryotype déséquilibré. Le type de ségrégation alternative, qui est le plus fréquent chez les oiseaux, conduit à la formation en proportions égales de gamètes normaux et de gamètes hétérozygotes équilibrés [14]. Malgré la présence des microchromosomes, la poule et la caille domestiques s'avèrent être des modèles remarquables pour la cytogénétique animale.

\section{La cytogénétique des gamètes}

L'étude des chromosomes des gamètes des espèces domestiques ne fait que démarrer. Chez le mâle, la première tentative de visualisation des chromosomes du spermatozoïde a été faite chez le porc par Creighton et al. [15]. $\mathrm{Au}$ Laboratoire de cytogénétique de Jouy, après avoir mis au point la technique de fécondation interspécifique porc/hamster chez l'animal normal, nous avons commencé l'étude des caryotypes des spermatozoïdes issus des sujets porteurs de deux translocations réciproques (figure 6).

A ce jour aucune étude n'a été entreprise sur les chromosomes de l'ovocyte des animaux d'élevage. Pourtant il serait très utile d'avoir un modèle animal car les $20 \%$ environ d'ovocytes a caryotype anormal publiés par plusieurs équipes chez l'homme [1] ont subi les effets d'une série de traitements techniques nécessaires pour la fécondation in vitro : traitement hormonal des femmes donneuses, contrôle aux ultrasons de la croissance folliculaire, etc. En plus, pour des raisons d'éthique, les ovocytes examinés sont seulement ceux qui ont subi un échec VI
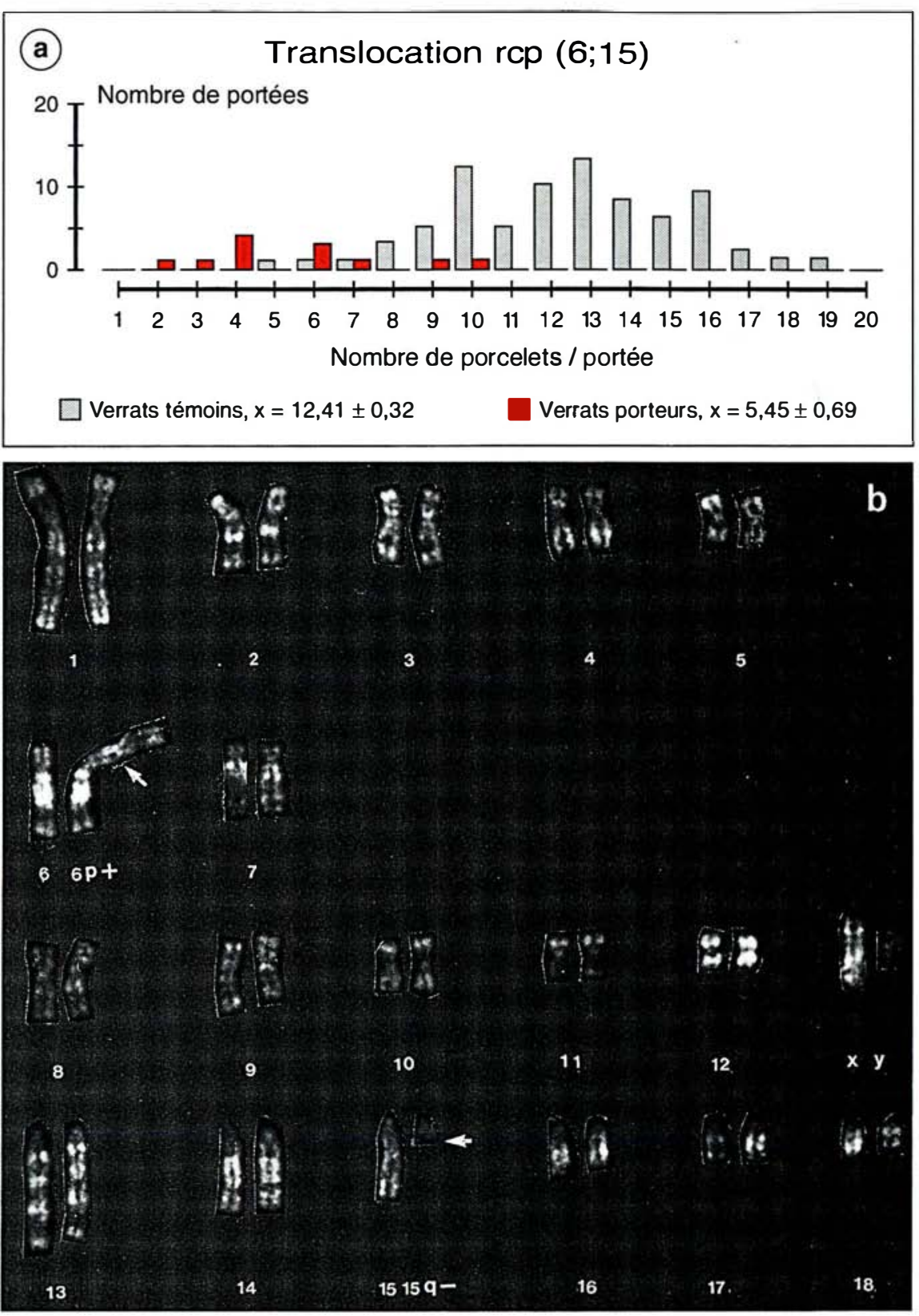

Figure 5. a. Histogramme des tailles de portées d'un verrat porteur de la translocation trcp $(6 ; 15)$ [5] Laboratoire de Cytogénétique-INRA-Jouy-en-Josas). b. Caryotype en bandes RBA du verrat porteur de la translocation trcp 16 ; 15) (J. Boscher, laboratoire de cytogénétique, INRA, Jouy-en-Josas).

\section{La carte génique}

La cartographie génétique comporte deux volets principaux : la carte génétique qui repère des distances en centimorgans entre des marqueurs polymorphes et la carte physique qui, elle, assigne les gènes sur les chromosomes. $\mathrm{Ce}$ dernier volet est l'affaire du cytogénéticien.

L'état actuel de la carte génique des animaux d'élevage est très peu avancé. Chez les bovins, 13 groupes de liaison ont été identifiés ainsi que environ 220 gènes et 30 fragments d'ADN 
appartenant a 31 groupes de synténie. La moitié des groupes de synténie et 5 groupes de liaison ont été assigné jusqu'à présent sur les chromosomes [16].

Chez le mouton 21 groupes de synténie ont été mis en évidence dont 7 ont été localisés. Une quarantaine de gènes ont été localisés sur les chromosomes. Chez la chèvre deux gènes ont été localisés et un groupe de liaison qui n'est pas encore assigné sur un chromosome donné.

Deux groupes de liaison sur 5 connus ont été localisé chez le cheval ainsi que 26 gènes. Chez le porc 3 groupes de liaison ont été assignés sur les 9 connus et environ 40 gènes localisés [16]. La connaissance du génome des espèces d'élevage connaîtra, dans les années à venir, un développement plus rapide que par le passé. Des collaborations internationales se mettent en place en Europe dans le cadre de la CEE et aux Etats-Unis [17, 18].

En ce qui concerne la carte physique des espèces de bovidés (vache, mouton et chèvre) et dans une moindre mesure celle du cheval, le problème crucial du travail de cartographie reste l'identification des chromosomes. En raison du nombre très élevé de leurs chromosomes et surtout de leur similitude morphologique on est confronté à des difficultés beaucoup plus grandes que celles posées, par exemple, par les chromosomes humains. En plus il existe actuellement très peu de laboratoires aussi bien en Europe qu'en Amérique, qui œuvrent sur les espèces domestiques et qui connaissent bien ces caryotypes. C'est pourquoi, l'amélioration de la qualité des préparations chromosomiques et des techniques de marquage qui permettent l'identification des chromosomes avec précision, devraient être les objectifs prioritaires des cytogénéticiens impliqués dans ces projets.

Les progrès qui seront accomplis dans la cartographie des animaux domestiques apporteront des outils précieux à la sélection animale et aux productions animales.

\section{Le tri des chromosomes}

La séparation physique des chromoso$m / s n^{\circ} 9$ vol. 7, novembre 91

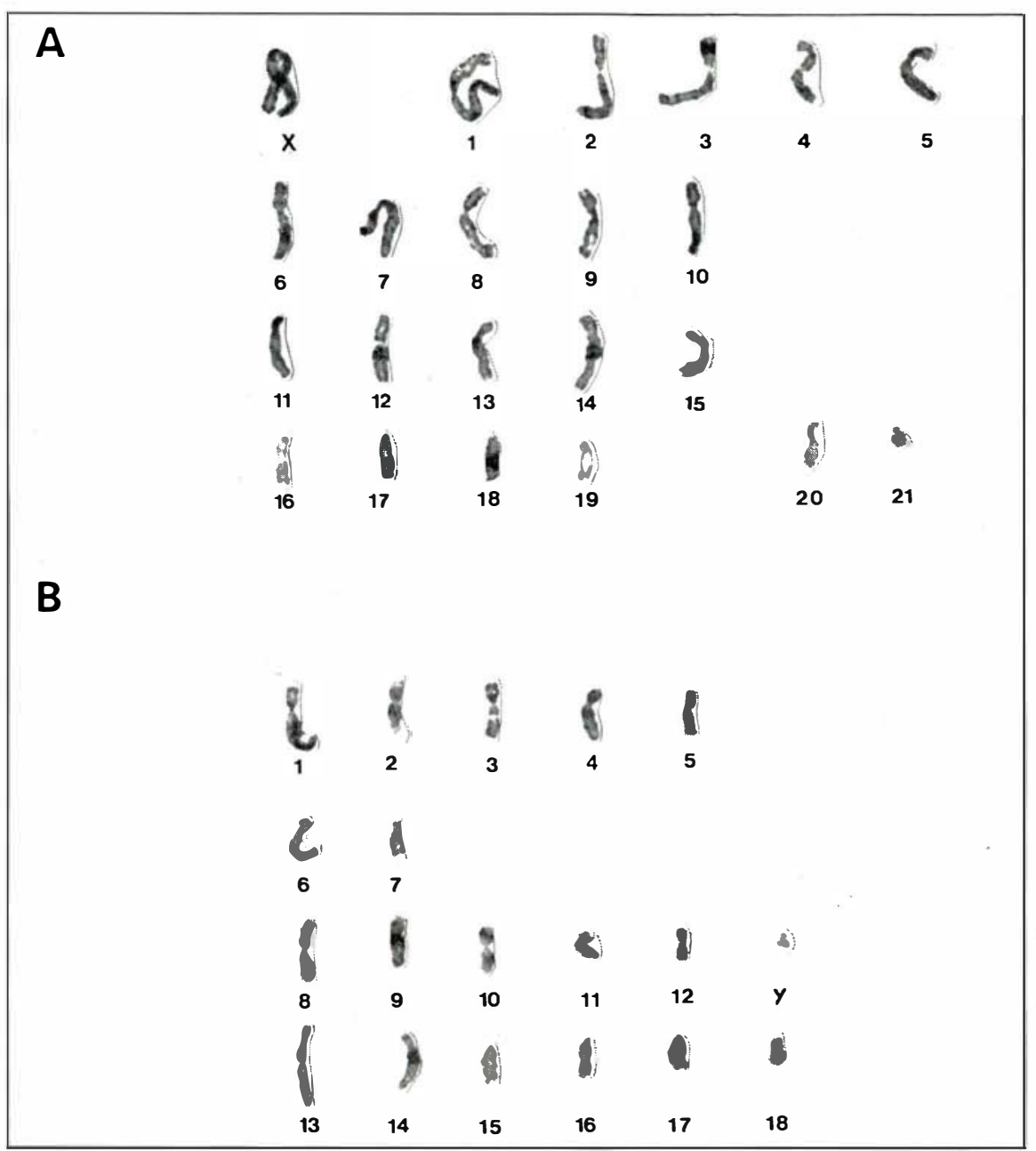

Figure 6. a Caryotype du spermatozoide de verrat et (b) d'ovocyte de hamster obtenus par fécondation in vitro $(M$. Benkalifa et $M$. Bonneau, laboratoire de cytogénétique, INRA, Jouy-en-Josas).

mes, appelée aussi le tri à l'aide de la cytométrie en flux, s'est beaucoup développée ces dernières années. En effet, pour l'approche de l'étude du génome des eucaryotes, il est indispensable d'effectuer un fractionnement préalable de l'ADN, par sonication ou action d'enzymes de restriction. Mais le tri des chromosomes est la seule technique de morcellement du génome qui conserve le chromosome intact.

Par la cytométrie en flux, on mesure la fluorescence individuelle des chromosomes qui passent à travers un faisceau laser (FACS-fluorescence activating cell sorter). La fluorescence des chromosomes étant proportionnelle à leur taille, ils donnent des signaux de fluorescence bien individualisés. Cela permet à un ordinateur de repérer un chromosome donné et de dévier la microgouttelette qui le contient dans un champ électrique. Elle sera ainsi dirigée vers une plaque déflectrice du condensateur et envoyée vers un récipient distinct (figure 7).

Le matériel utilisé est une suspension de chromosomes très bien dispersée. Cette suspension est injectée sous pression dans une cellule d'écoulement de $60 \mu \mathrm{m}$ de diamètre. Le flux de chromosomes d'un diamètre de $12 \mu \mathrm{m}$ est enveloppé d'une gaine liquide dont l'écoulement est laminaire, vers la zone de détection. La pression de l'échantillon de la gaine et la concen- 
tration en chromosomes, sont contrôlées de sorte que les chromosomes passent un à un dans le faisceau laser. La fluorescence des chromosomes est mesurée au moyen de détecteurs qui fournissent une tension électrique proportionnelle à l'intensité de la lumière reçue. Les impulsions électriques émises par les détecteurs sont filtrées et amplifiées. Ces signaux analogiques sont ensuite convertis en signaux numériques et présentés sous forme d'histogrammes de fréquence mono ou biparamétriques.

Sur un histogramme monoparamétrique (figure 8) les chromosomes sont séparés en plusieurs pics plus ou moins bien individualisés. La comparaison des niveaux de fluorescences et des longueurs relatives peut permettre d'estimer la position de certains chromosomes dans les pics. Dans un cas idéal un pic correspond à un seul chromosome, mais généralement dans un pic sont situés deux ou plusieurs chromosomes de taille similaire. L'utilisation de deux laser associée a une coloration avec deux fluorochromes augmente la résolution en flux des caryotypes en flux.

Chez l'homme, la cytométrie en flux adaptée à l'analyse et au tri des chromosomes est un outil très performant. Elle est utilisée pour la constitution des banques d'ADN, le dépistage des anomalies et polymorphismes chromosomiques et pour la cartographie génique.

Chez les animaux domestiques, seuls les chromosomes du porc ont été étudiés par la cytométrie en flux. Certaines anomalies chromosomiques, telles les translocations réciproques, peuvent être utilisées pour l'identification des chromosomes dans un caryotype en flux. En effet, les chromosomes remaniés, ayant la taille modifiée peuvent réduire ou augmenter certains pics ou encore en créer des nouveaux, ce qui permet d'établir une corrélation entre un pic donné et un chromosome particulier (figure 9) [19].

Dans l'avenir, la cytométrie en flux deviendra probablement une méthode de choix pour la construction de banques d'ADN et l'analyse du génome.

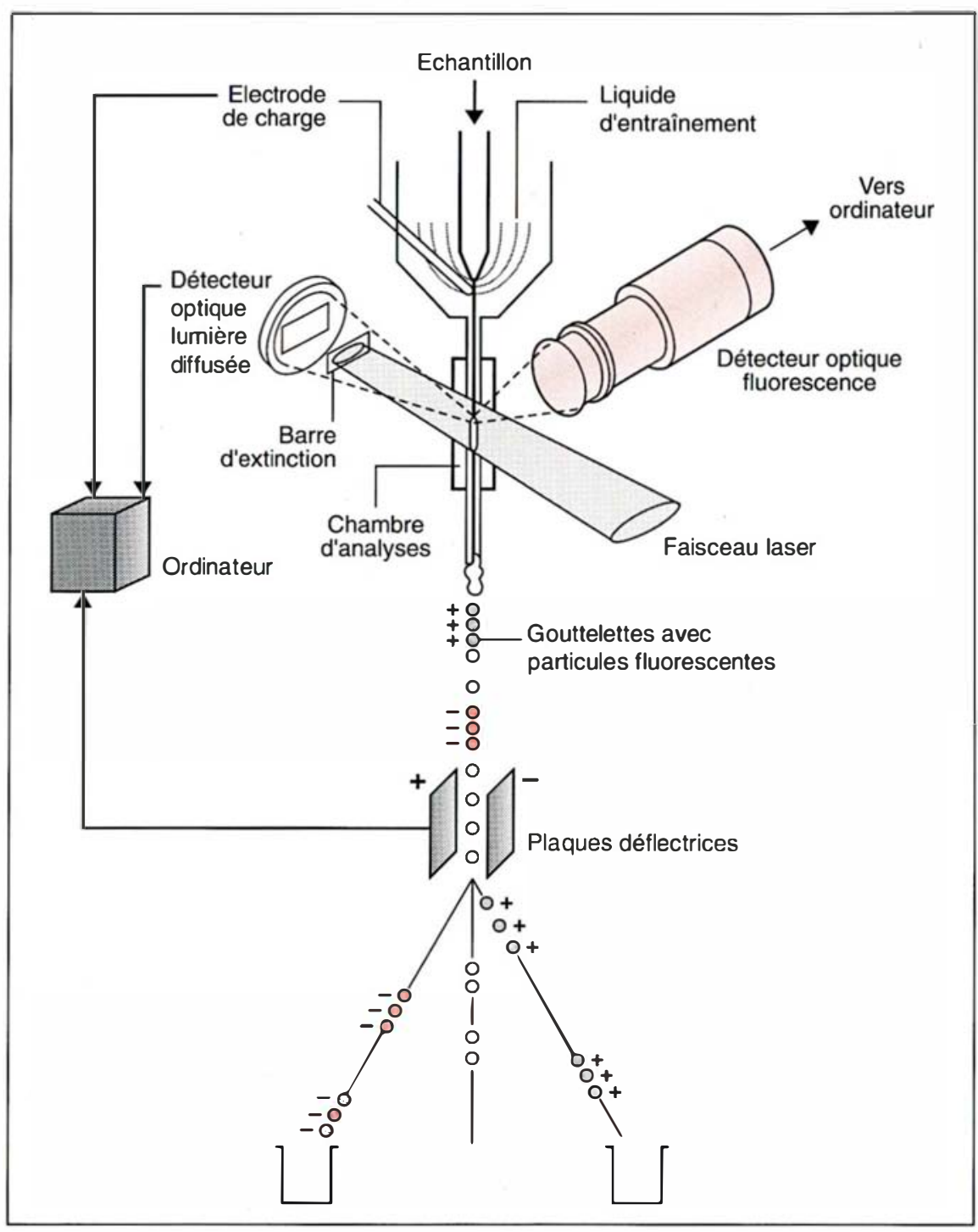

Figure 7. Schéma du trieur de chromosomes.

\section{Conclusion et perspectives}

"Putting the genetics back into cytogenetics". Ainsi intitulait le Dr. M. A. Fergusson-Smith, à qui la cytogénétique humaine doit beaucoup, un éditorial récent de la revue American Joumal of Human Genetics. Il considère, en effet, qu'en raison des développements récents et des nouvelles techniques dont s'est dotée la cytogénétique, cette discipline revient à juste titre au premier plan de la génétique humaine. Selon Fergusson-Smith, deux domaines de la cytogénétique ont revêtu une importance croissante ces dernières années et lui ouvrent des perspectives nouvelles. Le premier concerne la technique d'hybridation in situ, et le deuxième la cytogénétique du noyau interphasique.

La technique d'hybridation in situ avec des sondes non-radioactives et détection par fluorescence a été utilisée, dans un premier temps, pour déceler des sondes de plus d'un kb, insérées dans un plasmide. Elle a été récemment développée par Lichter et al. [20] sous le nom de "suppression des chromosomes in situ" (CISS) pour localiser de grands fragments génomiques 


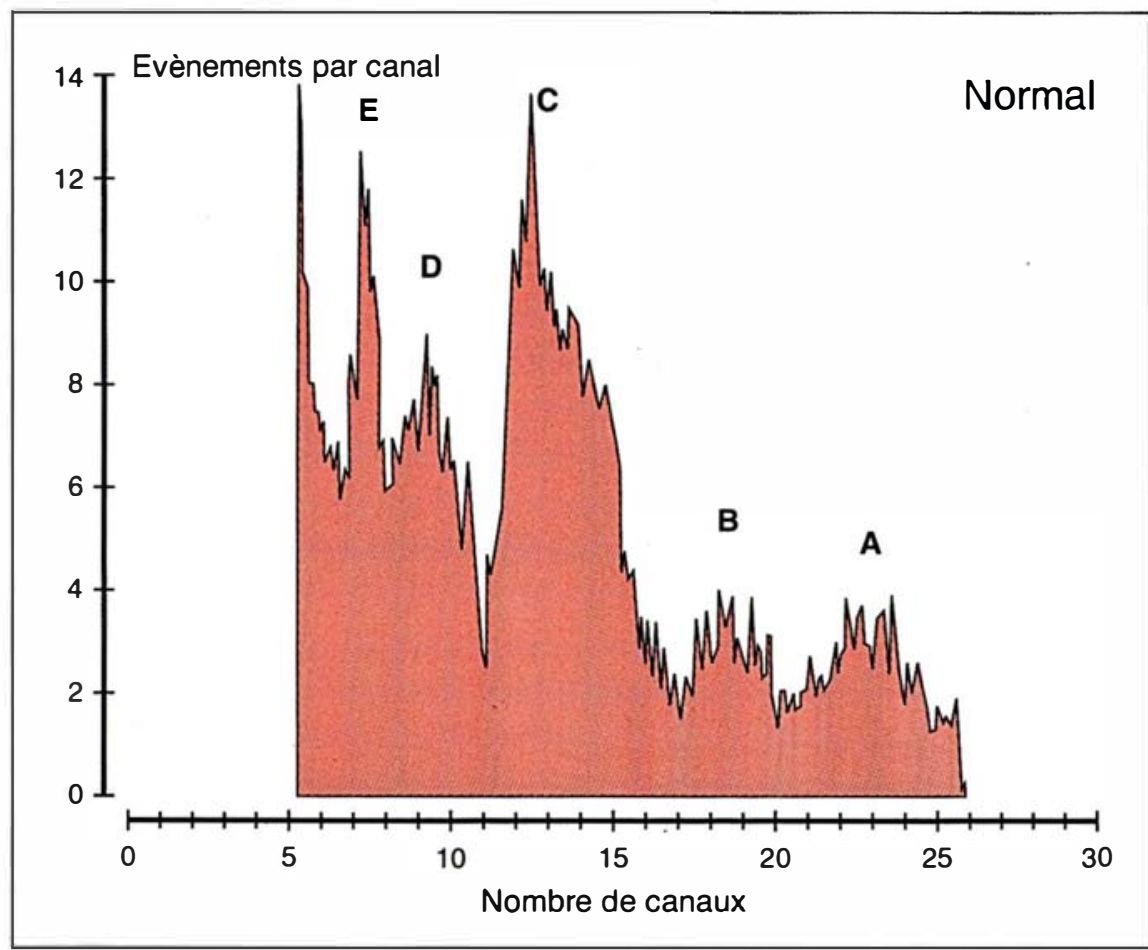

Figure 8. Caryotype en flux du porc normal. Les lettres $A$ à $E$ indiquent les pics de fluorescence. (D'après [18]).

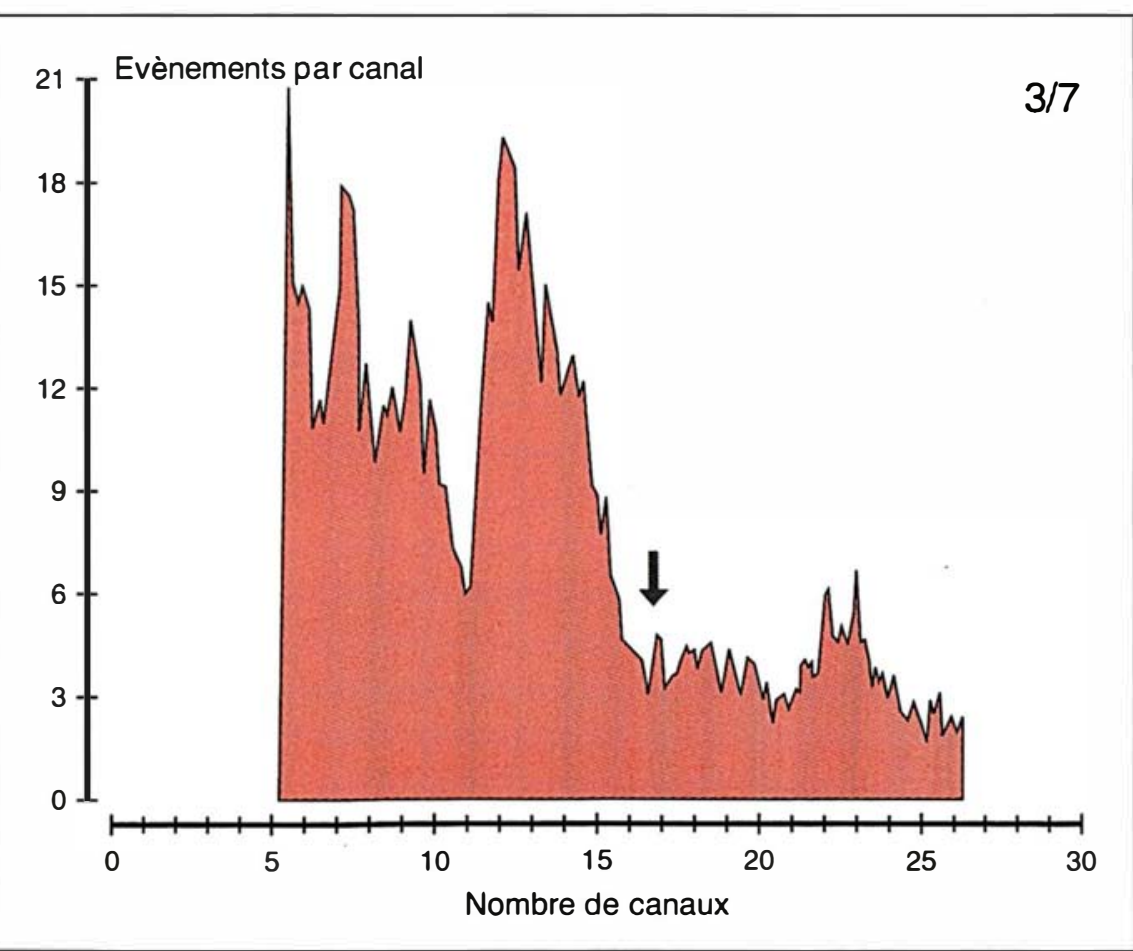

Figure 9. Caryotype en flux d'un verrat porteur de la translocation 3/7. La flèche indique un pic correspondant au chromosome remanié 3/7. (D'après [19]. insérés dans des cosmides ou des YAC (yeast artificial chromosomes). Dans ce cas les grandes séquences contiennent de nombreuses séquences répétées du type Alu ou Kpn, qui doivent être saturées avec de l'ADN génomique total nonmarqué. Ainsi les séquences répétées sont réassociées, permettant une hybridation sélective d'une séquence unique, avec les chromomes. Plusieurs sondes différentes peuvent être utilisées, chacune colorée avec un fluorochrome différent. Cela permet de localiser les sondes les unes par rapport aux autres et de les positionner par rapport au centromère et au télomère d'un chromosome.

L'hybridation avec des sondes d'un chromosome donné après la suppression des séquences répétées, permet de " décorer" (chromosome painting) un chromosome spécifique. Cette technique facilite beaucoup la détection des anomalies chromosomiques et s'avère très utile pour le diagnostic prénatal.

Les signaux d'hybridation in situ sont aussi visibles sur des noyaux interphasiques, ce qui permet d'analyser les relations spatiales entre des chromosomes à un stade ou ils ne sont pas optiquement visibles. Les chromosomes à l'interphase sont, en général 10 fois plus despiralisés que dans un noyau en métaphase, ce qui permet de positionner des sondes qui sont trop proches dans un noyau au stade de la métaphase. Ainsi, des séquences espacées de $50 \mathrm{kpb}$ peuvent être localisées ce qui augmentent la résolution de 20 fois, par rapport aux chromosomes métaphasiques [21].

L'application de ces techniques performantes aux chromosomes des animaux domestiques permettra de progresser rapidement dans la connaissance de leur génome et l'établissement de la carte physique. L'utilisation d'une banque de cosmides spécifiques pour chaque chromosome et d'une batterie de fluorochromes de couleurs différentes, pourrait résoudre les problèmes d'identification des chromosomes des bovidés. Elle facilitera la découverte des remaniements même les plus fins et la localisation des gènes avec un haut degré de résolution. 
La cytogénétique pourra également apporter sa contribution dans les travaux de transgénèse. Par hybridation in situ sur des chromosomes des animaux transgéniques, l'on cherchera à identifier le site d'intégration du gène réintroduit. On saura ainsi si l'intégration se fait à un site précis ou au hasard et on pourra suivre la transmission du gène réintroduit dans la descendance de l'animal transgénique. On pourra probablement mieux comprendre l'intéraction du gène réintroduit avec les autres gènes proches du site d'intégration et son expression.

Dans un avenir proche, la cytogénétique des animaux domestiques, contribura à améliorer nos méthodes de sélection et participera, avec les autres branches de la génétique animales, aux progrès des biotechnologies

\section{Références}

1. Pellestor F. Étude cytogénétique de l'ovocyte humain. médecine/sciences $1990 ; 6$ : 966-71.

2. Popescu CP. Cytogénétique des mammifères d'élevage. INRA, 1989: 114.

3. Legault C. Analyse des composantes de la productivité numérique des truies. Ann Zootech $1978 ; 27: 457-70$

4. Ford CE, Pollock DL, Gustavsson E. Proceedings of the first international conference for the standardization of banded karyotype of domestic animals, university of Reading, Reading, England, 2-6 august, 1976. Hereditas $1980 ; 92: 145-62$.

5. ISCNDA. International system for cytogenetic nomenclature of domestic animals (1989). In : Di Berardino D, Hayes H, Fries R, Long S, eds. Cytogenet Cell Genet 1990 ; $53: 65-79$.

6. Hayes H, Petit E, Dutrillaux B. Comparison of RBG-banded karyotypes of cattle, sheep and goats. Cytogenet Cell Genet 1991; $57: 51-5$.
7. Standard karyotype of the domestic horse. Equus caballus. Hereditas $1990 ; 112$ : 289-93.

8. Standard karyotype of the domestic pig Hereditas 1988 ; 109: 151-7.

9. Gustavsson I, Rockbom G. Chromosome abnormality in three cases of lymphatic leukaemia in cattle. Nature 1964 ; a : 203-990.

10. Frebling J, Foulley JL, Berland HM, Popescu CP, Cribiu EP, Darre R, 1987. Résultats de l'enquête sur la fréquence de la translocation $1 / 29$ en race bovine Blonde d'Aquitaine. Bull Tech CRZV Theix INRA 1987 ; 67 : 49-58.

11. Popescu CP, Tixier M. L'incidence des anomalies chromosomiques chez les animaux de ferme et leurs conséquences économiques. Ann Genet 1984 ; 27 : 69-72.

12. Bonneau M. Translocations réciproques. Conséquences chez le porc domestique. Thèse de diplôme de recherche de l'université Paris XI, 1990.

13. Chourrout S. Gynogenèse, polyplö̈die et transfert de gènes chez la truite arc-en-ciel (Salmo Gairdneri). Thèse de doctorat de l'université Paris VI, 1989.

14. Fechheimer NS. Chromosomes of chickens. In : Mc Feely, ed. Domestic Animal Cytogenetics. London : Academic Press, 1990.

15. Creighton P, Houghton JA. Visualization of pig sperm chromosomes by in vitro penetration of zona-free hamster ova. I Reprod Fert $1987 ; 80: 619-22$.

16. Chowdhary BP. Chromosomal mapping of some genes in farm animals using in situ hydridization. Thèse université d'Uppsala, 1991.

17. Haley CS, Archibald A, Andersson L, et al. The pig gene mapping project PiGMaP. Proceedings of the 4th world congress on genetics applied to livestosk production. 1990 ; XIII : $67-70$

18. Gellin J, Grosclaude E. Analyse du génome des espèces d'élevage : Projet d'établissement de la carte génique du porc et des bovins. INRA Prod Anim 1991; 4 : 97-105.

19. Blaise F, Aycardi J, Popescu CP. Flow cytometry of normal and abnormal pig karyotypes. Preliminary report. Ann Genel $1990 ; 33$ : 146-51.

20. Lichter P, Cremer T, Borden J, Manuelidis L, Ward DC. Delineation of individual human chromosomes in metaphase and interphase cells by in situ suppression hybridization using recombinant DNA libraries. Hum Gend $1988 ; 80$ : 224-34.

21. Trask BJ. Fluorescence in situ hybridization : applications in cytogenetics and gene mapping. Trends Gene 1991; 7 : 149-54.

\section{* GLOSSAIRE \\ Aneuploïde : une cellule qui ne contient pas le mêrne nombre de répétitions pour chaque chromosome. Cette situation peut être due à la perte ou, au contraire, à l'addition d'un ou de plusieurs chromosomes.}

Caryotype : les chromosomes d'une seule cellulle rangés dans une série décroissante d'après leur taille et la position du centromètre.

Chromosome métacentrique : chromosome ayant un centromère médian.

Chromosome submétacentrique : chromosome ayant le centromère situé dans la région submédiane.

Chromosome acrocentrique : chromosome ayant le centromère situé dans la région terminale.

Chromosome télocentrique : chromosome ayant un centromère terminal.

Gynogenèse : développernent d'un ouf activé par un spermatozoïde, mais sans la participation du génome mâle.

Isochromosome : chromosome possédant des bras égaux et identiques du point de vue génétique, attachés en position symétrique à un centromère central.

Longueur relative : la longueur réelle d'un chromosome rapportée à la longueur totale de tous les chromosomes d'une cellule et exprimée en pour mille.

Nombre fondamental (NF) : le nombre des bras d'un caryotype, indépendamment du nombre et de la forme des chromosomes.

Série robertsonienne : espèces apparentées qui possèdent le même nombre fondamental mais un nombre différent de chromosomes à cause d'une translocation robertsonienne.

P. Popescu : directeur du laboratoire de cytogénétique. INRA, CRJ, laboratoire de cytogénétique, 78352 Jouy-en-Josas Cedex, France. $m / s n^{\circ} 9$ vol. 7, rovembre 9 